\title{
Consumo de álcool durante a pandemia da COVID-19: uma reflexão necessária para o enfrentamento da situação
}

\author{
Alcohol consumption during the COVID-19 \\ pandemic: a necessary reflection for \\ confronting the situation
}

\section{Consumo de alcohol durante la pandemia de la COVID-19: una reflexión necesaria para \\ enfrentar la situación}

Leila Posenato Garcia 1

Zila M. Sanchez 2

doi: 10.1590/0102-311X00124520
O enfrentamento à pandemia da COVID-19 tem requerido, até o momento, a implementação de intervenções de Saúde Pública não farmacológicas, especialmente aquelas que visam ao distanciamento físico 1,2. Diferentes estratégias foram adotadas nos países afetados, desde o isolamento de casos e contatos até o bloqueio total (lockdown), incluindo a proibição do funcionamento de bares, restaurantes, casas noturnas, entre outros 3. Devido à restrição do funcionamento de tais estabelecimentos, o consumo de álcool, que era realizado em espaços públicos, passou ao privado, tendo o domicílio se tornado o local de escolha para esse comportamento.

Destaca-se que o consumo de bebidas alcoólicas está vinculado a mais de 230 doenças e agravos, como resultado dos efeitos do etanol, que é substância psicoativa, reforçadora, cancerígena, imunossupressora, tóxica para células e tecidos e teratogênica. É uma das principais causas de mortalidade evitável no mundo e responsável por 3 milhões de mortes a cada ano 4,5,6. A Organização Mundial da Saúde (OMS) considera que não existe limite seguro para o consumo do álcool e que o dano à saúde aumenta com a quantidade consumida 4,7. Especialmente, o consumo excessivo enfraquece o sistema imunológico e diminui a capacidade de o organismo combater as doenças infecciosas bacterianas $8 \mathrm{e}$ virais ${ }^{9}$, como a COVID-19, o que pode elevar o risco de infecção durante a pandemia.

Não obstante, no início da pandemia, circularam notícias falsas de que beber álcool forneceria alguma proteção contra a COVID-19 ou mataria o coronavírus. No Irã, por acreditarem nessas notícias, mais de 700 pessoas morreram após tomarem álcool de origem desconhecida e contaminado com metanol 10. Casos semelhantes foram reportados na Costa Rica e na República Dominicana. Visando proteger a população desse tipo de informação equivocada, a OMS lançou um informe no qual adverte que o consumo de álcool absolutamente não protege contra a COVID-19, ao contrário, pode prejudicar a resposta imunológica 5 .

Diversas hipóteses têm sido levantadas buscando discutir o efeito do álcool na saúde mental durante a pandemia 7. Em todas elas, destaca-se que o álcool é substância depressora do sistema nervoso central, e seu consumo é fortemente associado a outros transtornos mentais. Durante o isolamento, essa associação é potencializada e pode desencadear ou exacerbar episódios depressivos e ansiosos, como também aumentar o risco de suicídio. Com o incremento do uso regular e da quantidade de álcool consumida, no longo prazo, haverá aumento da tolerância e da dependência. Os potenciais efeitos na saúde pública do isolamento em longo prazo no consumo e uso indevido de álcool ainda são desconhecidos 6 . Não obstante, estudos realizados em diversos países apontaram maior ocorrência
1 Instituto de Pesquisa Econômica Aplicada, Brasília, Brasil. 2 Escola Paulista de Medicina, Universidade Federal de São Paulo, São Paulo, Brasil.

Correspondência L. P. Garcia Instituto de Pesquisa Econômica Aplicada. SBS Quadra 1 Bloco J, Brasília, DF 70076-900, Brasil.

leila.garcia@ipea.gov.br 
de ansiedade, depressão e consumo de álcool, além de menor bem-estar mental durante o isolamento como resposta à epidemia 11,12,13,14.

Entre jovens e adolescentes, tais efeitos podem levar alguns anos para serem identificados. Não se conhecem ainda as consequências para crianças que convivem com adultos que passaram a beber em casa, mas conjectura-se que essa exposição exacerbada esteja associada à iniciação precoce, pela facilidade do acesso, pela percepção de aceitação social do consumo 15 e pela vivência de mais episódios de violência doméstica 16 . Cabe destacar que, entre adolescentes, os estilos parentais são associados ao padrão de consumo de álcool e supõe-se que a permanência em casa sob supervisão dos pais pode levar à diminuição do consumo, particularmente quando os pais adotam o estilo autoritário, ou seja, oferecendo mais monitoramento e responsividade às necessidades dos adolescentes 17 . Por outro lado, há que se considerar que pais que aumentaram seu consumo de álcool dentro de casa durante a quarentena, bebendo em maior frequência ou quantidade, contribuem para a alteração das crenças normativas de seus filhos, que podem passar a interpretar o beber como algo cotidiano. Na prática, essa alteração de crenças normativas está associada a padrões mais danosos de uso de álcool na adolescência e deve ser evitada 18,19. Ainda, é preciso considerar que uma parte dos pais que ampliou seu consumo pode ter entendido que era o momento de "ensinar seus filhos a beber", contrariando todo o conhecimento científico sobre a prevenção ao uso de álcool por menores de idade 20.

A busca pelo uso de álcool em situações de estresse ocorre, equivocadamente, por seu efeito depressor do sistema nervoso central, que, em uma primeira fase, parece relaxar quem o consumiu. Por outro lado, esse mesmo efeito farmacológico é responsável pela ocorrência de diversos tipos de acidentes. Quedas, queimaduras e choques, assim como acidentes de trânsito, podem causar lesões que, por vezes, requerem atendimentos de urgência 21. No contexto da pandemia, os acidentes que geram lesões que requeiram atendimento, além de sobrecarregarem os serviços, podem elevar o risco de transmissão da COVID-19 nos próprios serviços de saúde.

Outro efeito negativo do consumo do álcool, potencializado pelo distanciamento físico, é a violência doméstica e familiar, cujas principais vítimas são mulheres e crianças 22,23 . No Brasil, entre os dias 1-16 e 17-25 de março de 2020, houve crescimento de $18 \%$ no número de denúncias registradas pelos serviços Disque 100 e Ligue 180 24. Também houve aumento no número de feminicídios em vários estados. Em São Paulo, esse número, em março de 2020, foi 46,2\% superior ao mesmo mês no ano anterior, enquanto, em Mato Grosso, os feminicídios quintuplicaram 25. Os homens são os principais perpetradores da violência doméstica e familiar contra mulheres, que é exacerbada pelo consumo de álcool. As sobreviventes, por sua vez, tendem a aumentar o uso de álcool como forma de lidar com a situação de violência 26 .

Ainda, há evidências de que o risco de dependência de álcool é maior à medida que as pessoas são mais expostas a desastres naturais. Um estudo longitudinal norte-americano identificou que tanto o número de furacões quanto o de eventos estressantes ocorridos na semana posterior foram preditores diretos das doses de álcool consumidas pelas pessoas expostas no ano seguinte 27. Comportamento semelhante foi encontrado após exposição a ataque terrorista 28 , reforçando a evidência de que a ocorrência de traumas ou de estresse pós-traumático aumenta o transtorno por uso de álcool 29.

Também há estudos que apontam intensificação do uso de álcool em situações de luto 30. Assim, à medida que a COVID-19 desponta como uma das principais causas de morte, surge a preocupação adicional sobre os padrões de consumo de álcool durante o isolamento e nos próximos anos. Sendo a pandemia uma vivência de potencial iminência de morte e por se assemelhar à experiência de episódios traumáticos naturais, é possível imaginar que os padrões de consumo de álcool posteriores serão aumentados, com implicações para a mortalidade e morbidade associadas.

No momento, tem sido observado internacionalmente aumento no consumo de álcool em casa, aparentemente decorrente do isolamento, e na contramão das evidências sobre os danos associados. $\mathrm{Na}$ China, 32\% dos consumidores habituais de álcool relataram aumento do uso, e 19\% relataram relapso ao abuso do álcool durante a pandemia ${ }^{31}$. No Reino Unido, quase um quinto dos participantes de uma pesquisa que relataram beber álcool diariamente aumentaram a quantidade consumida durante o lockdown 32 . Na Alemanha, 34,7\% dos respondentes de uma pesquisa on-line reportaram que passaram a beber mais ou muito mais álcool após o início do lockdown 33. No Brasil, a situação não é diferente. Pesquisa on-line realizada com 44.062 participantes revelou que $18 \%$ da população com 18 anos ou mais de idade relataram aumento do uso de bebidas alcoólicas durante a pandemia ${ }^{34}$. Levan- 
tamento encomendado pela Associação Brasileira de Bebidas (Abrabe) 35, em 2019, revelou que 61\% do consumo de bebidas alcoólicas acontecia em bares, restaurantes, casas noturnas e eventos diversos. Com o fechamento desses estabelecimentos e a proibição da aglomeração de pessoas, o consumo passou a ser realizado predominantemente no ambiente doméstico, incentivado também por eventos on-line, como happy hours e festas, que incitaram o aumento das vendas de bebidas em supermercados.

Cabe ressaltar que a ida ao supermercado é uma situação de potencial exposição ao coronavírus e que o risco de contágio pode ser aumentado quando a pessoa está sob o efeito do álcool. A redução da percepção de risco decorrente de seu efeito farmacológico ${ }^{36}$ pode resultar em diminuição na adesão às medidas preventivas entre os usuários, inclusive a interrupção do isolamento para a aquisição e consumo de bebidas alcoólicas, na não adesão às medidas de higiene e no uso incorreto das máscaras.

No Brasil, a regulação da venda de bebidas alcoólicas em supermercados é quase inexistente. Nesses locais, é permitido expor bebidas em áreas de grande visibilidade, inclusive próximo aos caixas, realizar promoções e vender embalagens com múltiplas unidades, sem limite de quantidade, nem restrição de dia da semana ou horário. Ainda, há facilidade de compra em sites e aplicativos, inclusive de bebidas geladas, sem contato com o comprador, como ocorre na Austrália 37. Nessas situações, o controle real da idade de quem recebe as bebidas é ausente. Esse cenário expõe a necessidade de reforço ao controle da venda para menores de idade, tanto na internet quanto nos pontos de venda físicos. A disponibilidade de bebidas nos supermercados e para entrega em domicílio facilita o consumo, o que vai de encontro às recomendações da estratégia SAFER da OMS 38, sobre redução da disponibilidade de bebidas alcoólicas, apresentada no Brasil em 2019.

Em outro extremo, em diversas partes do mundo, autoridades proibiram temporariamente a venda de bebidas alcoólicas, sob justificativas que incluem a necessidade da redução do uso de leitos de terapia intensiva para traumas e liberação dessas vagas para o tratamento de pessoas com COVID-19, manter a ordem social e proteger mulheres e crianças da violência doméstica. São exemplos: a África do Sul, a cidade de Nuuk (capital da Groenlândia), a cidade de Bancoque (capital da Tailândia), a região de Aisne (Norte da França), e o estado da Pensilvânia (Estados Unidos). Na América Latina, Bolívia, Panamá, e alguns estados do México também proibiram, enquanto outros países limitaram muito a compra para estocar em casa, como a Colômbia. O governo do México 39 endossou a posição da OMS para restrições de acesso ao álcool 40.

Alguns municípios do Brasil, assim como os estados do Piauí e do Paraná, decretaram lei seca temporária visando reforçar as medidas de isolamento. Embora possam ser consideradas radicais, tais medidas reforçam a mensagem de que o consumo de álcool não é essencial para a população e de que o sistema de licenciamento para venda de bebidas alcoólicas deve ser revisado, de modo que o Estado assuma maior responsabilidade pela proteção das pessoas contra os danos associados ao consumo doméstico, tanto no contexto da pandemia como no futuro 41,42 .

A inclusão do enfrentamento ao álcool como parte da resposta à COVID-19 justifica-se mediante a constatação de que se trata de fator de risco para a própria infecção, seja por um mecanismo biológico 43, seja pela capacidade de potencializar a aglomeração de pessoas em eventos de lazer 44 e aumentar a demanda hospitalar devido aos traumas decorrentes da intoxicação alcoólica. Nesse contexto, é urgente que os governos e as entidades civis do campo da saúde adotem estratégias para orientar a população sobre os danos associados ao consumo de álcool, disseminando a posição da OMS. Ademais, a regulação sobre o uso e a comercialização de bebidas alcoólicas deve ser reforçada, de maneira alinhada aos interesses da Saúde Pública e à estratégia SAFER. Tais ações devem ser complementadas pela manutenção e fortalecimento dos serviços de apoio aos usuários de álcool e outras drogas. 


\section{Colaboradores}

L. P. Garcia e Z. M. Sanchez participaram da concepção do artigo, da revisão da literatura, da redação e da revisão final; estão de acordo com a versão submetida para publicação e assumem responsabilidade pela integridade do artigo.

\section{Informações adicionais}

ORCID: Leila Posenato Garcia (0000-0003-11462641); Zila M. Sanchez (0000-0002-7427-7956).

\section{Agradecimentos}

À Dra. Maristela G. Monteiro que, com seu vasto conhecimento sobre álcool e políticas públicas, aportou valiosas contribuições para a elaboração deste artigo.

\section{Referências}

1. Garcia LP, Duarte E. Intervenções não farmacológicas para o enfrentamento à epidemia da COVID-19 no Brasil. Epidemiol Serv Saúde 2020; 29:e2020222.

2. Qualls N, Levitt A, Kanade N, Wright-Jegede N, Dopson S, Biggerstaff M, et al. Community mitigation guidelines to prevent pandemic influenza - United States, 2017. MMWR Recomm Rep 2017; 66:1-34.

3. Petersen E, Wasserman S, Lee SS, Go U, Holmes AH, Abri SA, et al. COVID-19: we urgently need to start developing an exit strategy. Int J Infect Dis 2020: 96:233-9.

4. World Health Organization. Global status report on alcohol and health 2018. https:// www.who.int/substance_abuse/publications/ global_alcohol_report/en/ (acessado em 28/ Abr/2020).

5. World Health Organization. Alcohol and COVID: what do you need to know? http:// www.euro.who.int/__data/assets/pdf_ file/0010/437608/Alcohol-and-COVID-19what-you-need-to-know.pdf?ua $=1$ (acessadoem 05/Mai/2020).

6. Clay JM, Parker MO. Alcohol use and misuse during the COVID-19 pandemic: a potential public health crisis? Lancet Public Health 2020; 5:e259.

7. Rehm J, Kilian C, Ferreira-Borges C, Jernigan D, Monteiro M, Parry CDH, et al. Alcohol use in times of the COVID 19: implications for monitoring and policy. Drug Alcohol Rev 2020; 39:301-4.

8. Gamble L, Mason CM, Nelson S. The effects of alcohol on immunity and bacterial infection in the lung. Med Mal Infect 2006; 36:72-7.
9. Szabo G, Aloman C, Polyak SJ, Weinman SA, Wands J, Zakhari S. Hepatitis C infection and alcohol use: a dangerous mix for the liver and antiviral immunity. Alcohol Clin Exp Res 2006; 30:709-19.

10. Shokoohi M, Nasiri N, Sharifi H, Baral S, Stranges S. A syndemic of COVID-19 and methanol poisoning in Iran: time for Iran to consider alcohol use as a public health challenge? Alcohol 2020; 87:25-7.

11. Ahmed MZ, Ahmed O, Aibao Z, Hanbin S, Siyu L, Ahmad A. Epidemic of COVID-19 in China and associated psychological problems. Asian J Psychiatr 2020; 51:102092.

12. Haider II, Tiwana F, Tahir SM. Impact of the COVID-19 pandemic on adult mental health. PakJ Med Sci 2020; 36(COVID19-S4):S90-4.

13. Verma S, Mishra A. Depression, anxiety, and stress and socio-demographic correlates among general Indian public during COVID-19. Int J Soc Psychiatry 2020; 66:756-62.

14. Smith L, Jacob L, Yakkundi A, McDermott D, Armstrong NC, Barnett Y, et al. Correlates of symptoms of anxiety and depression and mental wellbeing associated with COVID-19: a cross-sectional study of UK-based respondents. Psychiatry Res 2020; 291:113138.

15. McCutcheon VV, Agrawal A, Kuo SI, Su J, Dick DM, Meyers JL, et al. Associations of parental alcohol use disorders and parental separation with offspring initiation of alcohol, cigarette and cannabis use and sexual debut in high-risk families. Addiction 2018; 113:336-45. 
16. Sharman SJ, Coomber K, Mayshak R, Curtis A, Hyder S, Walker A, et al. Situational characteristics uniquely associated with children's exposure to intimate partner violence. J Interpers Violence 2019; (Online ahead of print).

17. Valente JY, Cogo-Moreira H, Sanchez ZM. Gradient of association between parenting styles and patterns of drug use in adolescence: a latent class analysis. Drug Alcohol Depend 2017; 180:272-8.

18. Sanchez ZM, Valente JY, Fidalgo TM, Leal AP, Medeiros PFP, Cogo-Moreira H. The role of normative beliefs in the mediation of a schoolbased drug prevention program: a secondary analysis of the \#Tamojunto cluster-randomized trial. PLoS One 2019; 14:e0208072.

19. Conegundes L, Valente JY, Cogo-Moreira H, Martins CB, Andreoni S, Sanchez ZM. Transition from nonuse to use of alcohol or binge drinking among adolescents: secondary analysis of a randomized controlled trial. Addict Behav 2020; 102:106159.

20. Gilligan C, Thompson K, Bourke J, Kypri K, Stockwell T. "Everybody else is doing it": norm perceptions among parents of adolescents. J Stud Alcohol Drugs 2014; 75:908-18.

21. Kaysen D, Dillworth TM, Simpson T, Waldrop A, Larimer ME, Resick PA. Domestic violence and alcohol use: trauma-related symptoms and motives for drinking. Addict Behav 2007; 32:1272-83.

22. van Gelder N, Peterman A, Potts A, O'Donnell M, Thompson K, Shah N, et al. COVID-19: reducing the risk of infection might increase the risk of intimate partner violence. EClinicalMedicine 2020; 21:100348.

23. World Health Organization. COVID-19 and violence against women: what the health sector/system can do? https://apps.who.int/iris/ bitstream/handle/10665/331699/WHO-SRH20.04-eng.pdf? ua $=1$ (acessado em 15/Mai/ 2020).

24. Ministério da Mulher, da Família e dos Direitos Humanos. Coronavírus: sobe o número de ligações para canal de denúncia de violência doméstica na quarentena. https://www.gov.br/ $\mathrm{mdh} / \mathrm{pt}-\mathrm{br} /$ assuntos/noticias/2020-2/marco/ coronavirus-sobe-o-numero-de-ligacoes-paracanal-de-denuncia-de-violencia-domestica-naquarentena (acessado em 28/Mar/2020).

25. Fórum Brasileiro de Segurança Pública. Violência doméstica durante pandemia de Covid-19. http://forumseguranca.org.br/publicacoes_ posts/violencia-domestica-durante-pandemiade-covid-19/ (acessado em 04/Mai/2020).

26. World Health Organization. Global and regional estimates of violence against women: prevalence and health effects of intimate partner violence and non-partner sexual violence. https://www.who.int/reproductivehealth/pub lications/violence/9789241564625/en/ (acessado em 04/Mai/2020).

27. Cerdá M, Tracy M, Galea S. A prospective population based study of changes in alcohol use and binge drinking after a mass traumatic event. Drug Alcohol Depend 2011; 115:1-8.
28. Hasin DS, Keyes KM, Hatzenbuehler ML, Aharonovich EA, Alderson D. Alcohol consumption and posttraumatic stress after exposure to terrorism: effects of proximity, loss, and psychiatric history. Am J Public Health 2007; 97:2268-75.

29. Gilpin NW, Weiner JL. Neurobiology of comorbid post-traumatic stress disorder and alcohol-use disorder. Genes Brain Behav 2017; 16:15-43.

30. Drabwell L, Eng J, Stevenson F, King M, Osborn D, Pitman A. Perceptions of the use of alcohol and drugs after sudden bereavement by unnatural causes: analysis of online qualitative data. Int J Environ Res Public Health 2020; 17:677.

31. Sun Y, Li Y, Bao Y, Meng S, Sun Y, Schumann $\mathrm{G}$, et al. Brief report: increased addictive internet and substance use behavior during the $\mathrm{CO}$ VID-19 pandemic in China. Am J Addict 2020; 29:268-70.

32. The Lancet Gastroenterology \& Hepatology. Drinking alone: COVID-19, lockdown, and alcohol-related harm. Lancet Gastroenterol Hepatol 2020; 5:625.

33. Koopmann A, Georgiadou E, Kiefer F, Hillemacher T. Did the general population in Germany drink more alcohol during the COVID-19 pandemic lockdown? Alcohol Alcohol 2020; (Online ahead of print).

34. Fundação Oswaldo Cruz. Resultados da ConVid: pesquisa de comportamentos. https:// convid.fiocruz.br/index.php?pag=bebiba_al coolica (acessado em 24/Jun/2020).

35. Bouças C. Faturamento do setor de bebidas alcoólicas cai 52\%. Valor Econômico 2020; 6 abr. https://valor.globo.com/empresas/noti cia/2020/04/06/faturamento-do-setor-de-be bidas-alcoolicas-cai-52 percent-com-covid-19. ghtml.

36. Gan G, Guevara A, Marxen M, Neumann M, Jünger E, Kobiella A, et al. Alcohol-induced impairment of inhibitory control is linked to attenuated brain responses in right fronto-temporal cortex. Biol Psychiatry 2014; 76:698-707.

37. Colbert S, Wilkinson C, Thornton L, Richmond R. COVID-19 and alcohol in Australia: industry changes and public health impacts. Drug Alcohol Rev 2020; (Epub ahead of print).

38. Monteiro MG. A iniciativa SAFER da Organização Mundial da Saúde e os desafios no Brasil para a redução do consumo nocivo de bebidas alcoólicas. Epidemiol Serv Saúde 2020; 29:e2020000.

39. Comisión Nacional contra las Adicciones. Consumo de bebidas alcohólicas durante la Jornada Nacional de Sana Distancia por COVID-19. https://www.gob.mx/salud/conadic/prensa/ consumo-de-bebidas-alcoholicas-durantela-jornada-nacional-de-sana-distancia-porcovid-19-241056 (acessado em 12/Mai/2020). 
40. World Health Organization. Alcohol does not protect against COVID-19: access should be restricted during lockdown. http://www.euro. who.int/en/health-topics/disease-prevention/ alcohol-use/news/news/2020/04/alcoholdoes-not-protect-against-covid-19-accessshould-be-restricted-during-lockdown/_rec ache? fbclid=IwAR2lkIQcxfDdziOa7esAbXOcaLv66wvhmP4JxoDwXQd5z14X02jAmHZulg (acessado em 12/Mai/2020).

41. Hobin E, Smith B. Is another public health crisis brewing beneath the COVID-19 pandemic? Can J Public Health 2020; 111:392-6.
42. Reynolds J, Wilkinson C. Accessibility of 'essential' alcohol in the time of COVID-19: casting light on the blind spots of licensing? Drug Alcohol Rev 2020; 39:305-8.

43. Testino G. Are patients with alcohol use disorders at increased risk for Covid-19 infection? Alcohol Alcohol 2020; 55:344-6.

44. Tutenges S, Bøhling F. Designing drunkenness: how pubs, bars and nightclubs increase alcohol sales. Int J Drug Policy 2019; 70:15-21. 